\title{
Fan fault diagnosis based on symmetrized dot pattern and improved BP neural network
}

\author{
Songling Wang $^{\mathrm{a}}$, Haixiao $\mathrm{Liu}^{\mathrm{b}}$ and Xiaogang $\mathrm{Xu}^{\mathrm{c}}$ \\ School of Energy Power and Mechanical Engineering, North China Electric Power University, \\ Baoding071003, China \\ aliuhxstar@163.com, bliuhxsunny@163.com, xxy@ncepu.edu.cn
}

\begin{abstract}
Keywords: Centrifugal fan, Fault diagnosis, Symmetrized dot pattern (SDP), Improved Back Propagation (BP) neural network
\end{abstract}

\begin{abstract}
To accurately diagnose the mechanical failure of fan, the method based on the symmetrized dot pattern (SDP) analysis and improved Back Propagation (BP) neural network is proposed. Vibration signals acquisition of 13 kinds of running states were achieved on the 4-73 No.8D centrifugal fan test rig and the SDP technique was utilized to reconstruct the vibration signals. Then, the features of the SDP pattern of each running state were extracted and the fault eigenvectors based on the multiple feature fusion were constructed. Finally, the sample set of the eigenvectors was trained and tested by the improved BP neural network to diagnose the mechanical failure of the fan. The results show that the fan fault diagnosis method based on the SDP analysis and improved BP neural network can effectively diagnose the category, severity and site of the fan mechanical failures with high diagnosis rate, short testing time and good online diagnosis performance.
\end{abstract}

\section{Introduction}

The running state of the fan has the direct bearing on the safe and economic operation of the power plant, and the reliability, security and economy of the fan depends on the efficient operation, the real-time status tracking evaluation, the accurate diagnosis and repair, thus it has great significance to launch the fault diagnosis research of the fan. There are lots of fault diagnosis methods, but basically divided into three steps: acquisition of the diagnostic information; fault feature extraction; state recognition and fault diagnosis.

Collecting the vibration signal is the most widely used method for fault monitoring and diagnosis currently. It is necessary to analyze the signals after sufficient vibration signals are collected. General signal analysis is mainly in the time domain, frequency domain and time-frequency domain. However, due to the vibration signal generally belong to the non-stationary signal and the signal of interest is low compared to the background noise, general signal feature extraction methods have a large limitation for the fan vibration signal. As a new signal processing method, the symmetrised dot pattern (SDP) analysis can fully describe the characteristic of signal and express it in visual graphics[1,2]. The technique is particularly effectively compared to other techniques when the signal of interest is low compared to the background noise.

\section{Experimental simulation of fan machinery fault diagnosis}

Different mechanical vibration experiments were done on the 4-73 No.8D centrifugal fan test bench of North China Electric Power University. The experiment selected the Schenck Germany IN-81 eddy current displacement probe and the PCB's Model 481 signal filter. Five eddy current sensors were mounted on both sides of the fan bearing. This paper simulates 13 different running states of the fan, which are shown in Table.1. Each measuring point measured 20 vibration signal samples in each state, that is, a total of $13 \times 5 \times 20$ vibration signal samples were collected. The sampling frequency is $1600 \mathrm{~Hz}$ and the speed of the fan is $1200 \mathrm{rpm}$. 
Tab.1 The fan running state of the simulation

\begin{tabular}{|c|c|c|}
\hline $\begin{array}{l}\text { The category label } \\
\text { of of each fan running } \\
\text { state } \\
\end{array}$ & $\begin{array}{l}\text { The fan running } \\
\text { state of the simulation }\end{array}$ & commentary \\
\hline 1 & Normal & \multirow{5}{*}{$\begin{array}{l}\text { The rotor unbalance fault was simulated } \\
\text { by installing the unbalanced mass on the six } \\
\text { uniform angle positions close to the outer edge } \\
\text { of the front disc. Different severity and } \\
\text { different positions of the rotor unbalanced fault } \\
\text { were simulated by adjusting the quality and } \\
\text { location of the unbalanced mass. }\end{array}$} \\
\hline 2 & Rotor unbalanced & \\
\hline 3 & Rotor unbalanced & \\
\hline 4 & Rotor unbalanced & \\
\hline 5 & Rotor unbalanced & \\
\hline 6 & Angular & \multirow{5}{*}{$\begin{array}{l}\text { Coupling of the bench is the rigid } \\
\text { coupling. The angular misalignment, parallel } \\
\text { misalignment (light) and parallel misalignment } \\
\text { (serious) of the coupling were simulated. }\end{array}$} \\
\hline & misalignment & \\
\hline 7 & $\begin{array}{c}\text { Parallel } \\
\text { misalignment (light) }\end{array}$ & \\
\hline 8 & Parallel & \\
\hline & misalignment (serious) & \\
\hline 9 & $\begin{array}{l}\text { Bearing loose (all } \\
\text { loose light) }\end{array}$ & \multirow{3}{*}{$\begin{array}{l}\text { Different severity and different positions } \\
\text { of the bearing loose were simulated by } \\
\text { adjusting the tightness of the bolts on the } \\
\text { different parts of the bearing. }\end{array}$} \\
\hline 10 & $\begin{array}{l}\text { Bearing loose (all } \\
\text { loose serious) }\end{array}$ & \\
\hline 11 & $\begin{array}{c}\text { Bearing loose (the } \\
\text { left loose) }\end{array}$ & \\
\hline 12 & $\begin{array}{l}\text { Static and kinetic } \\
\text { friction (1) }\end{array}$ & \multirow{2}{*}{$\begin{array}{l}\text { The varying degrees of the static and } \\
\text { kinetic friction of the single point, multi-point } \\
\text { and local face were simulated. }\end{array}$} \\
\hline 13 & $\begin{array}{l}\text { Static and kinetic } \\
\text { friction (2) }\end{array}$ & \\
\hline
\end{tabular}

\section{The Method of SDP Analysis}

The SDP plotting technique is shown in Figure 1.

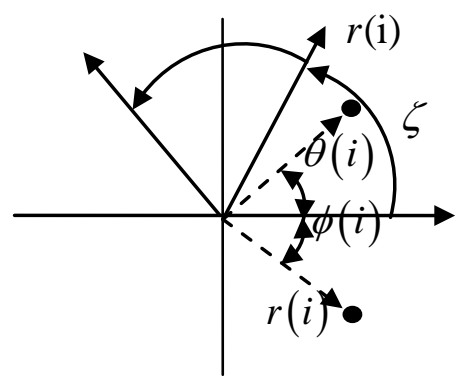

Fig. 1 Principle of SDP method

A point in the time waveform maps onto a radial component and the adjacent point maps to an angular component. We can formulate the polar transformation $r(i)$ from waveform to SDP as

$$
\begin{array}{r}
r(i)=\frac{x_{i}-x_{\min }}{x_{\text {max }}-x_{\min }} \\
\theta(i)=\theta+\frac{x_{i+l}-x_{\min }}{x_{\text {max }}-x_{\min }} \zeta \\
\phi(i)=\theta-\frac{x_{i+l}-x_{\min }}{x_{\text {max }}-x_{\min }} \zeta
\end{array}
$$

where: 
$i$ is the number of dot ( $i=\operatorname{integer}(t / \Delta t))$, with $t$ the time abscissa and $\Delta \mathrm{t}$ the sampling time;

$l$ is the time lag coefficient;

$x_{i}$ is the sampled $i$ th sound signal;

$x_{\max }$ and $x_{\min }$ are the highest and the lowest value of the original waveform window;

$n$ is the number of mirror symmetry plane;

$\theta$ is the rotation of the origin angle of any reference line $\left(\theta=360^{\circ} \mathrm{m} / \mathrm{n}, \mathrm{m}=1,2, \mathrm{~L}, n\right)$;

$\zeta$ is the gain of the plotting $(\zeta \leq \theta)$;

$\theta(i)$ and $\phi(i)$ are the two angles of the traditional polar space.

\section{SDP Patterns of Vibration Signals of Fan}

Take $\theta=360^{\circ} / 5, \zeta=30^{\circ}$ and $l=1$, the vibration signals of 13 kinds of fan running states, shown in Table 1, were analyzed by the SDP technique. Parts of the SDP patterns are shown in Figure 2.

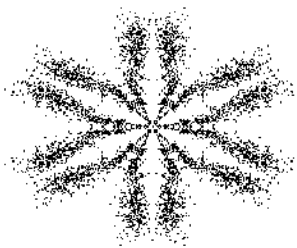

Normal

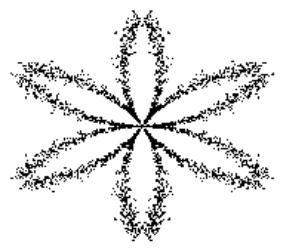

Rotor unbalanced (1)

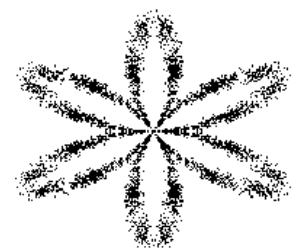

Bearing loose (all loose serious)

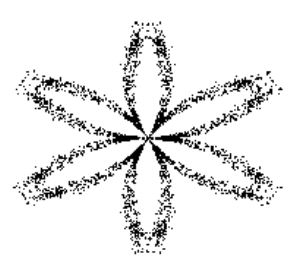

Rotor unbalanced (1)

Fig. 2 Parts of the SDP patterns of the fan.

\section{Extract the features of the SDP pattern}

According to the value of radius, the SDP pattern is divided into ten regions, shown in figure 3.

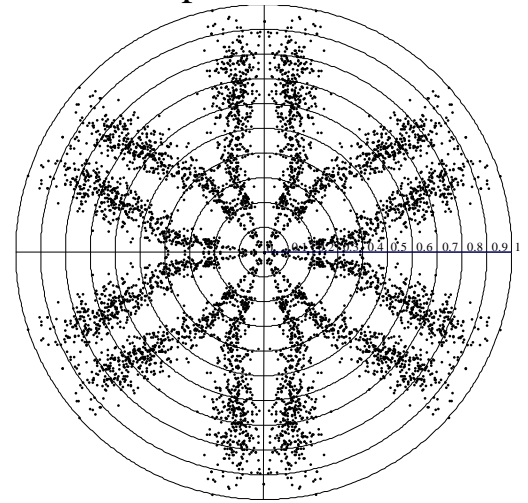

Fig. 3 The dividing areas of the SDP pattern

Extract the percentage of the number about points in the SDP arms in each region as the first feature, expressed by $E_{1}$. Extract the variances of $r(i)$ about points in the SDP arms in each region as the second feature and the variances of $\theta(i)$ about points in the SDP arms in each region as the third feature, expressed by $E_{2}$ and $E_{3}$. Finally, the fault eigenvectors based on the multiple feature fusion were constructed, expressed by $E=\left[E_{1}, E_{2}, E_{3}\right]$.

Based on the actual data of vibration, the fault eigenvectors of the $13 \times 5 \times 20$ signals were extraced and fault eigenvectors of 260 samples in 13 different conditions were got.

\section{Fault diagnosis based on the SDP analysis and improved BP neural network}

The improved BP neural network was utilized in this paper, whose neuron number of the input layer, hidden layer and output layer were 30, 65 and 13. Through training and testing, the results show that the diagnostic accuracy of the network is the highest when the transfer function of the 
input layer neurons and the hidden layer neurons, the hidden layer neurons and the output layer neurons are tansig(), logsig(). The neural network training method with the momentum term and the adaptive learning rate was utilized based on the supervised learning [3, 4]. The expected error minimum value of the network was le-5. The maximum number of iteration was 2000 . The learning rate was 0.01 . The Momentum factor was 0.90 .

Select 130 samples of 13 different states from the 260 samples as the training samples; and the remaining 130 sets of fault eigenvectors are used as test samples to input the improved BP neural network for diagnosis. the accuracy rate of fault diagnosis is 100\%, shown in Figure 4.

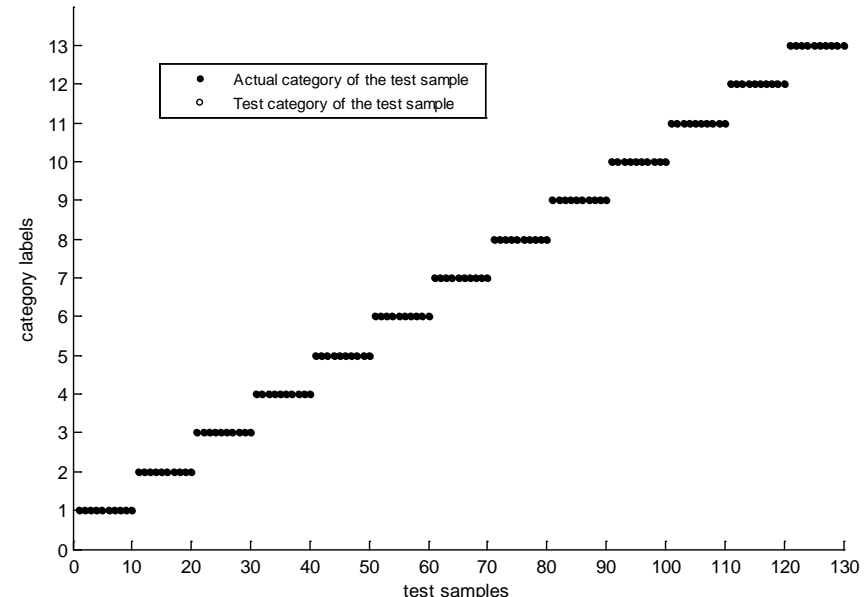

Fig. 4 Fault diagnosis result based on the SDP analysis and improved BP neural network

\section{Summary}

The fan fault diagnosis method based on the SDP analysis and improved BP neural network can effectively diagnose the category, severity and site of the fan mechanical failures with high diagnosis rate, short testing time and good online diagnosis performance.

\section{References}

[1] Sheard A G, Corsini A, Bianchi S. Stall warning in a low-speed axial fan by visualization of sound signals [J]. Journal of Engineering for Gas Turbines and Power. Vol 133 (2011) No. 4, p. 041601-041610.

[2] Stefano B, Alessandro C, Anthony GS. Demonstration of a stall detection system for induced draft fans. Journal of Power and Energy. Vol 227 (2013) No. 3, p. 272-284.

[3] Wang Songling, Xu Xiaogang, Liu Jinlian, et al. Fault diagnosis of fan based on symbolic dynamics entropy and improved neural network [J]. Journal of North China Electric Power University. Vol 40 (2013) No. 4, p. 51-58.

[4] Mi Jiang, Ji Guoyi. Application of Improved BP Neural Network in Fault Diagnosis of Fans [J]. Noise and Vibration Control. Vol 31 (2011), No. 2, p. 94-98. 